\title{
Resonance in Magnetostatically Coupled Transverse Domain Walls
}

\author{
A. T. Galkiewicz, ${ }^{1}$ L. O'Brien, ${ }^{2,3}$ P. S. Keatley, ${ }^{4}$ R. P. Cowburn, ${ }^{3}$ and P. A. Crowell ${ }^{1, *}$ \\ ${ }^{1}$ School of Physics and Astronomy, University of Minnesota, \\ 116 Church St. SE, Minneapolis, MN 55455 \\ ${ }^{2}$ Department of Chemical Engineering and Materials Science, \\ University of Minnesota, 421 Washington Ave. SE, Minneapolis, MN 55455 \\ ${ }^{3}$ Thin Film Magnetism Group, Cavendish Laboratory, \\ University of Cambridge, JJ Thomson Ave, \\ Cambridge CBз OHE, United Kingdom \\ ${ }^{4}$ School of Physics, University of Exeter, \\ Stocker Road, Exeter EX4 4QL, United Kingdom
}

\begin{abstract}
We have observed the eigenmodes of coupled transverse domain walls in a pair of ferromagnetic nanowires. Although the pair is coupled magnetostatically, its spectrum is determined by a combination of pinning by edge roughness and dipolar coupling of the two walls. Because the corresponding energy scales are comparable, the coupling can be observed only at the smallest wire separations. A model of the coupled wall dynamics reproduces the experiment quantitatively, allowing for comparisons with the estimated pinning and domain wall coupling energies. The results have significant implications for the dynamics of devices based on coupled domain walls.
\end{abstract}

PACS numbers: $75.60 . \mathrm{Ch}, 75.75-\mathrm{c}, 75.78-\mathrm{n}, 85.70 . \mathrm{Kh}$ 
Interactions between nano-scale ferromagnetic domains or domain walls (DWs) can become significant as separations approach the dimensions of the individual structures. These interactions can modify existing resonant behavior [1, 2], lead to the appearance of new cou-

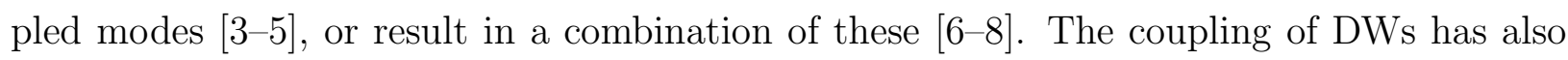
been proposed as a scheme towards increasing the output power of spin-torque oscillators [9], and will have an impact on the design of DW-logic-based devices as well. Understanding resonant behavior in these and similar systems is thus of great interest, as resonances provide a direct probe of the underlying coupling. The system of two interacting transverse domain walls (TDWs) in parallel nanowires (NWs) [Fig. 17(b)] is one of the simplest manifestations of coupled DWs. Despite great interest in this particular coupling both experimentally and numerically [4, 5, 10], no experimental observation of the coupled mode has yet been observed.

In this paper, we report on the observation of the resonant excitation of coupled TDWs in adjacent NWs. By comparing such coupled excitations with those from single TDWs and micromagnetic simulations, we show the experimental results may only be understood with the inclusion of intrinsic pinning due to NW roughness: an effect predominantly neglected when considering dynamic DW excitation. Extending simple one-dimensional (1D) analytical models to describe phenomenologically both inter-DW coupling and intrinsic DW pinning reproduces the observed spectra and provides insight into the interaction. The frequency of one of the two modes of the coupled wall system is determined almost entirely by the pinning energies of the individual walls, while only the second mode depends significantly on the magnetotstatic coupling. We demonstrate that intrinsic pinning and DW coupling remain comparable even at small inter-wire separations; therefore pinning through roughness must always be considered when investigating such dynamical DW experiments.

Figure 1(a) shows a scanning electron microscope (SEM) image of the sample geometry. Pairs of Permalloy semicircular NWs with radii of $5 \mu \mathrm{m}$ and thickness of $10 \mathrm{~nm}$ were studied for NW widths $w$ of 70, 85, 140, and $190 \mathrm{~nm}$, and closest separations $d$ in the range of 40-140 nm. This design allows for repeatable DW nucleation in the region of closest NW separation (here onwards referred to as the interaction region) by the application of a saturation field along the $y$-direction $\mid 11 \|$. Based on the thickness and widths of the NWs, the stable configuration is a TDW [12, 13]. This is also seen in Fig. 1(b) using micromagnetic simulations [14] with a cell size of $2 \times 2 \times 10 \mathrm{~nm}^{3}$, saturation magnetization $M_{s}=800 \mathrm{kA} / \mathrm{m}$, 

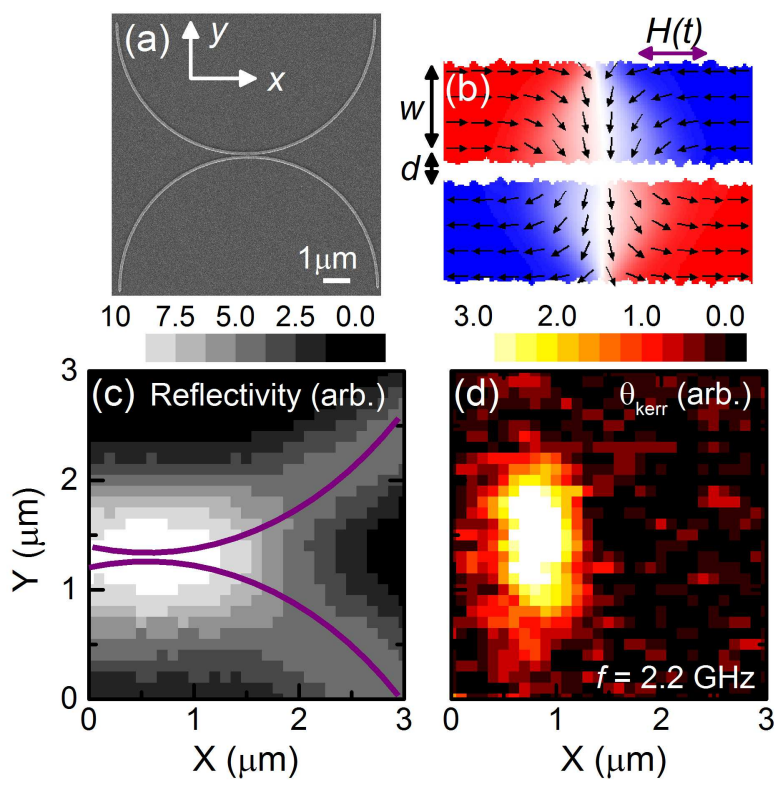

FIG. 1. (color online) (a) Scanning electron micrograph of a $w=85 \mathrm{~nm}, d=40 \mathrm{~nm}$ device. (b) Micromagnetic simulation of two coupled DWs in a $w=100 \mathrm{~nm}, d=20 \mathrm{~nm}$ pair of nanowires. Arrows indicate the in-plane direction of $\mathbf{M}$, and colors the magnitude of $M_{x}\left(+M_{s}\right.$ red, $-M_{s}$ blue). (c) 2D optical reflectivity map and (d) Kerr rotation map of a $w=85 \mathrm{~nm}, d=130 \mathrm{~nm}$ device. The solid lines in (c) are the nanowire positions. The Kerr rotation map is shown for the maximum response on resonance.

exchange constant $A=13 \mathrm{pJ} / \mathrm{m}$, and Gilbert damping $\alpha=0.01$.

The basis of our measurement is time-resolved Kerr microscopy, which utilizes the phaselocking of a microwave synthesizer (Agilent N5183A) to the $76 \mathrm{MHz}$ repetition rate of a pulsed Ti:Sapphire laser. The in-plane microwave field generated by the synthesizer drives the motion of the DWs, which are probed stroboscopically by focusing the pulse train (spot size of $\sim 400 \mathrm{~nm}$ ) onto the interaction region using an oil immersion objective. Through the use of the polar Kerr effect, we are sensitive to the net out-of-pane component of the dynamic magnetization [15]. Figure 1(c) shows the spatial map of the sample reflectivity localized to the interaction region of a $w=85 \mathrm{~nm}, d=130 \mathrm{~nm}$ pair of NWs. The solid lines show the NW positions. Figure1(d) - acquired simultaneously with Fig. 1(c) — shows the corresponding map of the $\theta_{\text {Kerr }}$ response for an on-resonance excitation. Clearly the excited response is localized in the interaction region, indicating that the observed spectra are due solely to the dynamic response of the DWs. 
We first investigate the response of a DW in a single NW. We expect, based on DW propagation experiments, that pinning from intrinsic defects such as edge or surface roughness will play a role in the dynamics [16, 17], although this has not been thoroughly explored for resonant dynamics. For vortex domain walls (VDWs) in NWs, studies have reported only on the characterization of a single intrinsic [18] or patterned [19] pinning site. Thus, it cannot be determined what influence intrinsic pinning (common to both cases) has on the resonant frequency. A similar situation exists for TDWs in NWs [20, 21], however in this case an intrinsically pinned TDW has not been investigated until now.

Figure 2(a) shows recorded spectra for isolated semicircular NWs that reveal DW resonances in the range of 1-2 GHz. The spectra are obtained by plotting the mean-squared amplitude of the time-resolved $M_{z}$ signals acquired for different frequencies of the driving Oersted field. As shown, different resonant frequencies are found when testing different NWs and when testing various DW nucleation sites in the same NW. These observations are suggestive of a pinned mode (PM), in which the DW oscillates in a local energy minimum created by the intrinsic roughness of the NW. The variations in frequency in such a case are due to the random distribution of pinning site depths and shapes. Figure 2(b) shows the average PM frequency of four devices at each width $w$. The error bars indicate the dispersion of observed frequencies. In the tested range of $w$, we see no dependence of the PM frequency on the width.

To confirm the origin of the PM, we perform dynamical micromagnetic simulations. A DW is prepared in a simulated NW, and a 6 Oe, 100 ps wide Gaussian field pulse is applied in the $x$-direction. The decay of the response is then recorded, and resonances are found by taking the Fourier transform of the volume-averaged $M_{z}(t)$. Edge effects from the simulation boundaries were determined to be negligible. We begin by simulating an ideal NW having zero roughness. As expected, the DW moves freely along the NW and no PM is observed. To include pinning, edge roughness, characterized by a root-mean-square roughness $\sigma_{r m s}$ and correlation length $\lambda$, is added to the NW. In these simulations, a PM is observed that is a collective oscillation of the DW spins, and results in the oscillating motion of the DW within the pinning site. From SEM images, we estimate $\sigma_{r m s}$ and $\lambda$ to be in the range of $1-4$ $\mathrm{nm}$ and 2-6 nm respectively. We find that the PM frequency is only weakly dependent on these two parameters, due to an effective convolution of the edge roughness with the larger DW profile $(\sim 100 \mathrm{~nm})$. Figure 2(b) shows the average PM frequency of six simulated NWs 

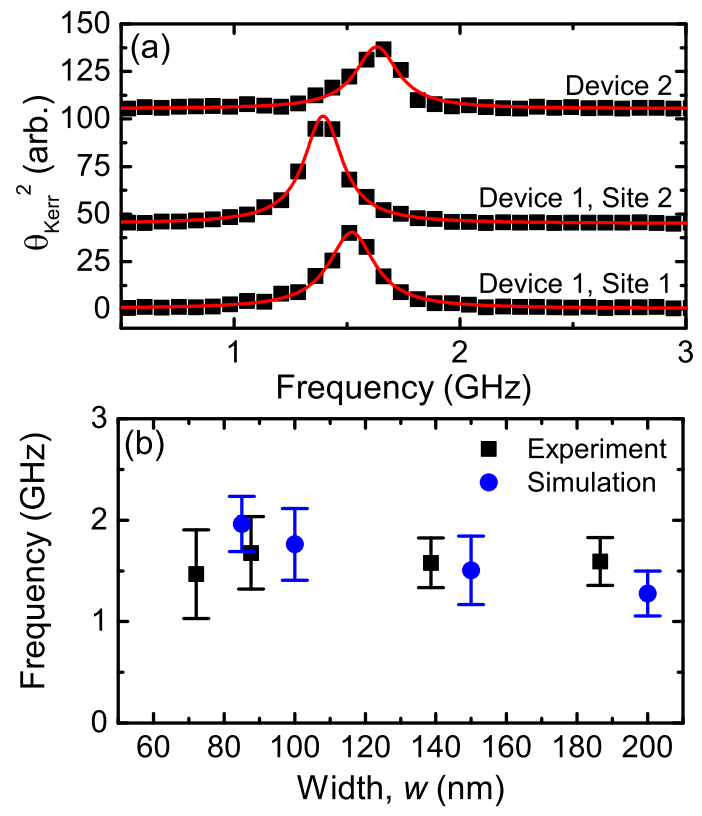

FIG. 2. (color online) (a) DW spectra for $w=85 \mathrm{~nm}$ nanowires taken at two DW nucleation sites and in a separate device. Spectra are offset vertically for clarity. The solid lines are Lorentzian fits from which the central frequency is extracted. (b) Averaged pinning site frequency as a function of nanowire width for experiment (squares) and micromagnetic simulation (circles). Error bars indicate dispersion of pinned mode frequencies over the range of tested devices in experiment and simulation.

versus width for $\sigma_{r m s}=2 \mathrm{~nm}$ and $\lambda=4 \mathrm{~nm}$, which match the data well. A slight decrease in the PM frequency with width is observed in simulation, which is the expected trend based on increases in the DW width and out-of-plane anisotropy. We have also observed modes internal to the DW in simulation that are characterized by a spatially non-uniform amplitude and phase [22], but due to their nonuniformity we expect a weak coupling to the optical probe.

We now turn our investigation to the case of coupled DWs. Resonant frequencies obtained from the measured spectra are plotted in Fig. 3)(a) for different separations $d$. As only slight $(<1 \mathrm{GHz})$ width dependence is expected [4] and no trend with respect to NW width is observed, we make no distinction in $w$ in this plot. Of the 43 devices tested, 20 show two peaks in their spectra and two devices show three peaks, the highest frequency of which are most likely internal modes. The inset of Fig. 3(a) is a sample spectrum that shows two modes corresponding to the two starred points. Also included in this plot is a hatched region 


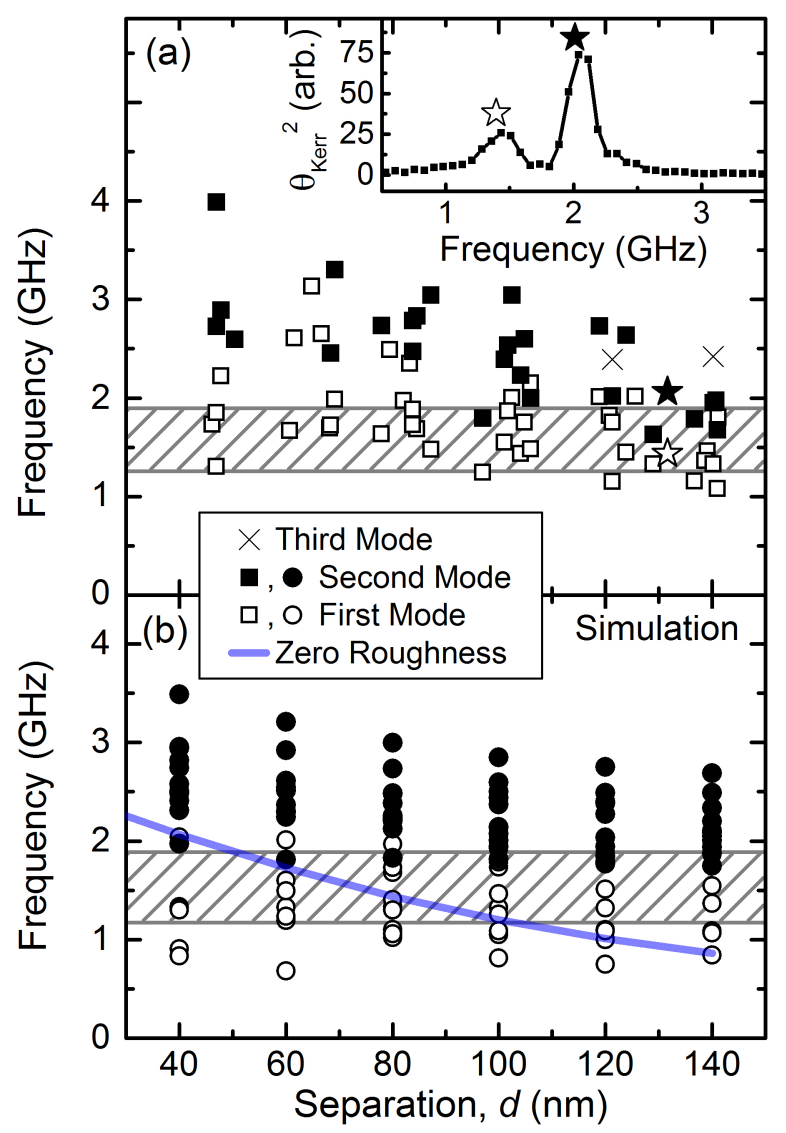

FIG. 3. (color online) Separation dependence of (a) experimental and (b) simulated coupled DW resonances. Open symbols correspond to the lowest frequency resonance observed in each spectrum, closed symbols correspond to the next lowest frequency, and a cross designates the two third modes seen in experiment. Data is included for all nanowire widths. Hatched regions in (a) and (b) are the average plus or minus one standard deviation of the single nanowire resonances from Fig. 2 , Inset in (a) is the spectrum for a $w=85 \mathrm{~nm}, d=130 \mathrm{~nm}$ nanowire pair with two resonances at 1.5 and $2 \mathrm{GHz}$. In (b) the solid line is the simulated DW-DW resonance for the case of a $w=100$ nm pair of nanowires with zero edge roughness.

that indicates the dispersion of PM frequencies from the single NW case. While we may statistically correlate the first mode with a PM, the frequency of the second mode is well above this region. In addition, the frequency of the second mode decreases with increasing separation, as would be expected for a mode dependent on the inter-DW coupling.

To further explore these modes, we utilize micromagnetics. We start by testing the case of zero edge roughness. In contrast to the zero-roughness single NW simulations, a translational 
mode is observed in the double NW system due to the inter-DW coupling. Figure 3 (b) shows the results of these simulations for a $w=100 \mathrm{~nm}$ pair, plotted as a solid line. Comparing to Fig. 33(a), the zero-roughness case cannot explain the higher frequencies and the observation of multiple modes. We thus proceed to test the system with the inclusion of edge roughness, using $\sigma_{r m s}=2 \mathrm{~nm}$ and $\lambda=4 \mathrm{~nm}$ as previously characterized from the single NW data. In these simulations the lowest two modes are translational, and plotting them gives the open and closed circles in Fig. 3(b), which shows good agreement with trends observed in experiment. Simulations also show a qualitative similarity in the phase response of the higher frequency mode compared to that of the zero-roughness DW-DW mode.

Given this quantitative agreement, we now look for an analytical understanding of this system using the $1 \mathrm{D}$ equations of motion [4, 23]. In these equations the DW is described by $x$ and $\phi$, the center coordinate and angle with respect to the $x y$ plane respectively. Letting $i=1$ or 2 denote the top or bottom DW, the equations of motion are

$$
\begin{aligned}
Q_{i} \frac{\dot{x}_{i}}{\Delta}-\alpha \dot{\phi}_{i} & =\frac{\gamma}{M_{s}} K_{s} \sin \left(2 \phi_{i}\right), \\
Q_{i} \dot{\phi}_{i}+\frac{\alpha}{\Delta} \dot{x}_{i} & =\frac{\gamma}{2 M_{s} S}\left[-\frac{\partial U_{i}}{\partial x_{i}}\right] .
\end{aligned}
$$

Here $Q_{1}=1, Q_{2}=-1$ are the effective magnetostatic charges of the two DWs, $\Delta$ is the DW width, $K_{s}$ is the out-of-plane anisotropy, $\gamma$ is the gyromagnetic ratio, and $S$ is the cross-sectional area of the nanowire. $U_{i}$ is the total energy of a DW and is given by $U_{i}=U_{0}-Q_{i} M_{s} S H x_{i}+k\left(x_{1}-x_{2}\right)^{2}+k_{i} x_{i}^{2}$, where the terms on the right hand side are the internal energy, Zeeman energy, interaction energy, and pinning energy due to roughness respectively. We have assumed a harmonic potential for the roughness. In the limit of small $\phi_{i}$, Eq. (1) can be diagonalized and the natural frequencies of the resulting two modes are given by

$$
\omega_{0}^{2}=\frac{k_{1}+k_{2}+2 k}{m_{D}} \pm \frac{\sqrt{\left(k_{1}-k_{2}\right)^{2}+(2 k)^{2}}}{m_{D}},
$$

where $m_{D}=\left(1+\alpha^{2}\right) M_{s}^{2} S / \gamma^{2} \Delta K_{s}$ is the DW Döring mass [24]. Figure 4(a) shows the simplified description of this system as a coupled oscillator problem in which each mass (DW) sits in its own potential (pinning site). We note here that an in-phase response of the DW displacements $x_{i}$ is accompanied by an out-of-phase response of $\phi_{i}$ and vice versa, due to the coupling of $x$ and $\phi$ in Eq. (11). We also note that given two dissimilar pinning site spring constants, both modes of Eq. (2) will couple to the driving magnetic field and 

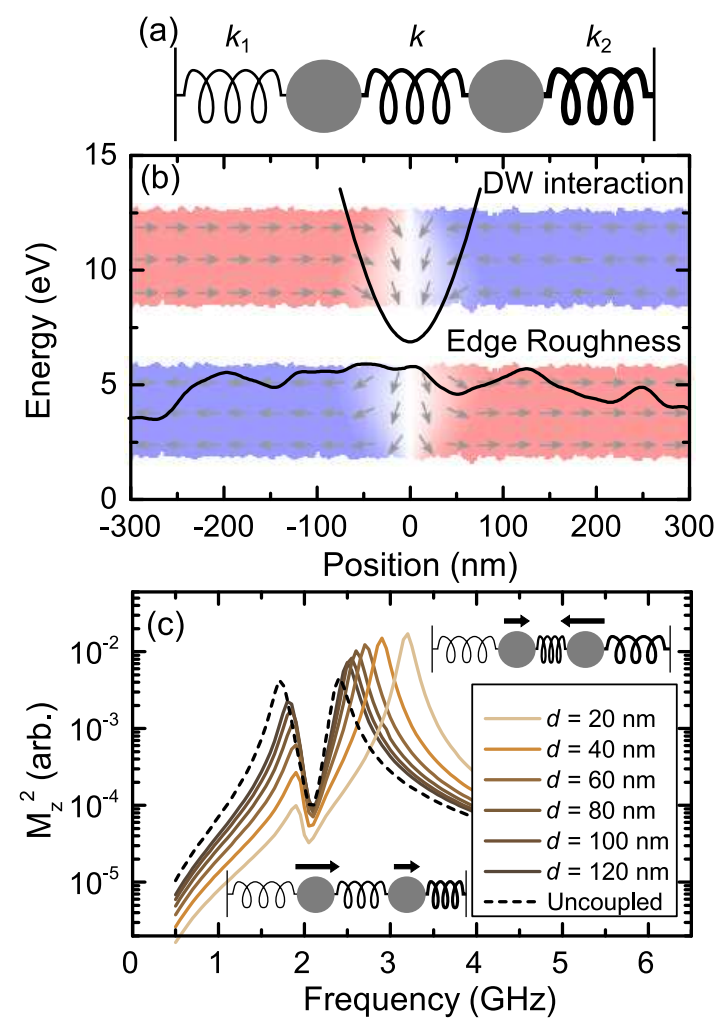

FIG. 4. (color online) (a) Simplified model of the coupled DWs: two oscillating masses coupled together with spring constant $k$. (b) Energy profiles for the edge roughness of a $w=100 \mathrm{~nm}$ nanowire (lower) and the DW interaction (upper) for a $w=100 \mathrm{~nm}, d=20 \mathrm{~nm}$ pair, as a function of the position of the DWs in the nanowires, offset for clarity. Nanowires shown in the background share the x-axis scale. (c) Numerically solved $M_{z}^{2}=\left(\sin \phi_{1}+\sin \phi_{2}\right)^{2}$ of the 1-D coupled DW equations of motion [Eq. (11)] as a function of drive frequency and plotted for multiple nanowire separations, shown for the case of two $w=100 \mathrm{~nm}$ nanowires. Values used were $k_{1}=0.21$ $\mathrm{erg} / \mathrm{cm}^{2}, k_{2}=0.40 \mathrm{erg} / \mathrm{cm}^{2}$, and $k=0.03-0.22 \mathrm{erg} / \mathrm{cm}^{2}$. Insets in bottom left (upper right) are representations of the lower (higher) frequency mode.

the relative oscillation amplitudes of the two DWs will be unequal. This enables both the in-phase and out-of-phase modes to be excited and detected experimentally. From Eq. (2), as $k$ goes to zero $(d \rightarrow \infty)$ the two modes approach the respective PM frequencies. As $k$ becomes large, the lower mode goes to the root-mean-square of the two PM frequencies, while the upper mode goes to the expected DW-DW resonance. Estimates of the spring constants can be found from energy landscapes calculated using micromagnetics which are illustrated in Fig. 4(b). To find the energy profile due to edge roughness, we translate a 
DW profile through the length of a NW and compute the total energy of the system at each position. We find the energy of the DW coupling by separating the two DWs laterally in zero-roughness NWs and computing the sum of the magnetostatic and exchange energies for different values of these separations. Note that the ranges of the DW interaction potential and a given pinning site are both set by the domain wall width. This is why the curvatures of the interaction potential and any given minimum of the magnetostatic potential due to edge roughness in Fig. 4(b) are comparable, in spite of their very different depths.

Using the estimates for the $k_{i}$ and $k$ obtained from micromagnetics, we numerically solve Eq. (11) and plot the power spectrum of the net $M_{z}$ component for multiple $d$ [Fig. 4(c)]. At large separations, the two DWs tend to their independent PM frequencies and phases. As $d$ decreases, the lower mode, illustrated in the bottom left inset, is suppressed due to the unfavorable driving force and the out-of-phase response of the $M_{z}$ components. These observations can explain the detection of only a single mode in some of the spectra: at large separations two accidentally degenerate PM frequencies will be unresolvable, while at small separations the lower frequency mode may be below the detection threshold. Looking at the upper mode (depicted in the top right inset) we see an increase in amplitude and frequency with decreasing $d$. The frequency dependence produced by this model agrees strongly with the trends observed in both micromagnetics and experiment. While experimental limitations such as non-ideal waveguides prevent a quantitative comparison of the relative mode amplitudes, in general a qualitative agreement is also seen [e.g. inset of Fig. 3(a)]. From these observations, it is clear that the first two modes in Fig. 31(a) can be attributed to the two eigenmodes of Eq. (2), given a random distribution of pinning sites in the NWs. As the width of this distribution is set in part by the DW itself, pinning will always be significant in the dynamics, and must be considered in future experiments.

This work was supported in part by the NSF MRSEC program under DMR-0804244 and the NSF/NRI NEB program under ECCS-1124831, as well as the EU Marie Curie IOF project no. 299376 and the European Community Seventh Framework Programme Contract No. 247368: 3SPIN. Parts of this work were carried out in the Characterization Facility, University of Minnesota, which receives partial support from NSF through the MRSEC program. 
* crowell@physics.umn.edu

[1] N. Kuhlmann, A. Vogel, and G. Meier, Phys. Rev. B 85, 014410 (2012)

[2] C. Swoboda, N. Kuhlmann, M. Martens, A. Vogel, and G. Meier, J. Appl. Phys. 114, 043905 (2013)

[3] R. L. Stamps, A. S. Carriço, and P. E. Wigen, Phys. Rev. B 55, 6473 (1997)

[4] L. O’Brien, E. R. Lewis, A. Fernández-Pacheco, D. Petit, R.P. Cowburn, J. Sampaio, and D. E. Read, Phys. Rev. Lett. 108, 187202 (2012).

[5] I. Purnama, M. Chandra Sekhar, S. Goolaup, and W. S. Lew, Appl. Phys. Lett. 99, 152501 (2011).

[6] J. Shibata, K. Shigeto, and Y. Otani, Phys. Rev. B 67, 224404 (2003)

[7] S. Jain, H. Schultheiss, O. Heinonen, F. Y. Fradin, J. E. Pearson, S. D. Bader, and V. Novosad, Phys. Rev. B. 86, 214418 (2012)

[8] A. Vogel, T. Kamionka, M. Martens, A. Drews, K. W. Chou, T. Tyliszczak, H. Stoll, B. Van Waeyenberge, and G. Meier, Phys. Rev. Lett. 106, 137201 (2011)

[9] A. Ruotolo, V. Cros, B. Georges, A. Dussaux, J. Grollier, C. Deranlot, R. Guillemet, K. Bouzehouane, S. Fusil, and A. Fert, Nat. Nanotechnol. 4, 528 (2009)

[10] L. OBrien, D. Petit, H. T. Zeng, E. R. Lewis, J. Sampaio, A. V. Jausovec, D. E. Read, and R. P. Cowburn, Phys. Rev. Lett. 103, 077206 (2009)

[11] E. Saitoh, H. Miyajima, T. Yamaoka, and G. Tatara, Nature 432, 203 (2004)

[12] M. Laufenberg, D. Bedau, H. Ehrke, M. Kläui, U. Rüdiger, D. Backes, L. J. Heyderman, F. Nolting, C. A. F. Vaz, J. A. C. Bland, T. Kasama, R. E. Dunin-Borkowski, S. Cherifi, A. Locatelli, and S. Heun, Appl. Phys. Lett. 88, 212510 (2006).

[13] Y. Nakatani, A. Thiaville, and J. Miltat, J. Magn. Magn. Mater. 290, 750 (2005).

[14] M. J. Donahue and D. G. Porter, Interagency Report NISTIR 6376 (National Institute of Standards and Technology, Gaithersburg, MD, 1999).

[15] T. Y. Chen and P. A. Crowell, IEEE Trans. Magn. 46, 1457 (2010).

[16] Y. Nakatani, A. Thiaville, and J. Miltat, Nat. Mater. 2, 521 (2003)

[17] E. Martinez, L. Lopez-Diaz, L. Torres, C. Tristan, and O. Alejos, Phys. Rev. B 75, 174409 (2007) 
[18] D. Bedau, M. Kläui, M. T. Hua, S. Krzyk, U. Rüdiger, G. Faini, and L. Vila, Phys. Rev. Lett. 101, $256602(2008)$

[19] R. Moriya, L. Thomas, M. Hayashi, Y. B. Bazaliy, C. Rettner, and S. S. P. Parkin, Nat. Phys. 4, $368(2008)$

[20] D. Bedau, M. Kläui, S. Krzyk, U. Rüdiger, G. Faini, and L. Vila, Phys. Rev. Lett. 99, 146601 (2007)

[21] L. J. Chang, P. Lin, and S. F. Lee, Appl. Phys. Lett. 101, 242404 (2012)

[22] X. Wang, G. Guo, G. Zhang, Y. Nie, Q. Xia, and Z. Li, J. Magn. Magn. Mater. 332, 56 (2013)

[23] A. Thiaville and N. Nakatani, in Spin Dynamics in Confined Magnetic Structures III (Springer, New York, 2006).

[24] W. Doring, Z. Naturforsch. A 3, 373 (1948). 